\title{
An Investigation of Scalar Dispersion in Grid Turbulence
}

\author{
P. E. Dimotakis ${ }^{1, *}$, D. B. Lang ${ }^{1}$, S. Lombeyda ${ }^{2}$, J. Lindheim ${ }^{2}$ \\ California Institute of Technology, Pasadena, California 91125, U.S.A \\ ${ }^{1}$ Graduate Aeronautical Laboratories \\ ${ }^{2}$ Center for Advanced Computing Research \\ Email: dimotakis@caltech.edu
}

\begin{abstract}
The structure of scalar dispersion from a continuous release point was investigated for moderate Reynolds number flow in grid turbulence. Using laser-induced fluorescence techniques, laser-volume scanning, a custom-designed fast-readout CCD focal plane array, and high-speed digital-imaging/acquisition/-storage techniques, the instantaneous three-dimensional structure of a passive scalar was investigated in flow in water (high Schmidt number). Laser Doppler and scalar-correlation velocimetry were employed to measure th flow speed entering the test section and in the interrogated volume. Such scalar-dispersion structure away from the release point is typically modeled assuming a Gaussian profile. This provides a good description for the mean scalar profile as a function of the transverse distance from a line parallel with the flow and downstream of the release point, as also confirmed by experiment (Yamamoto \& Sato 1979, Gad-el-Hak \& Morton 1979, Nakamura et al. 1987, Sawford 2001). The instantaneous three-dimensional structure, however, reveals a rich topology of scalar structures that was found to persist in the volume interrogated, spanning a distance from the grid and release point between 22 and 30 grid mesh lengths, which is in the self-similar grid-turbulence regime where the present three-dimensional scalar-field measurements were conducted.
\end{abstract}

\section{REFERENCES}

1. Yamamoto K, Sato Y. Measurements of Lagrangian behaviors of turbulent fluid. Study of Fundamental Engineering for Materials in Yokohama National University, 1979; 14: 25-36

2. Gad-el-Hak M, Morton JB. Experiments on the diffusion of smoke in isotropic turbulent flow. AIAA J., 1979; 17: 558-562

3. Nakamura I, Sakai Y, Miyata M. Diffusion of matter by a non-buoyant plume in grid turbulence. J. Fluid Mech, 1987; 178: 379-403

4. Sawford B. Turbulent relative dispersion. Ann. Rev. Fluid Mech., 2001; 33: 289-317 